\title{
QUASI-DIFFUSION SOLUTION OF A STOCHASTIC DIFFERENTIAL EQUATION
}

Abstract. We consider the stochastic differential equation

$$
X_{t}=X_{0}+\int_{0}^{t}\left(A_{s}+B_{s} X_{s}\right) d s+\int_{0}^{t} C_{s} d Y_{s},
$$

where $A_{t}, B_{t}, C_{t}$ are nonrandom continuous functions of $t, X_{0}$ is an initial random variable, $Y=\left(Y_{t}, t \geq 0\right)$ is a Gaussian process and $X_{0}, Y$ are independent. We give the form of the solution $\left(X_{t}\right)$ to $(0.1)$ and then basing on the results of Plucińska [Teor. Veroyatnost. i Primenen. 25 (1980)] we prove that $\left(X_{t}\right)$ is a quasi-diffusion proces.

1. Stochastic integral. In this section, we define stochastic integrals

$$
\int_{a}^{b} g(t) d_{t} Y(t, \omega)
$$

following [2] (see also [1] and [6]) in a way that will be useful in Section 2. Here $g: \mathbb{R} \rightarrow \mathbb{R}$ is a deterministic continuous function and $Y$ is a Gaussian process with an absolutely continuous covariance function. For abbreviation, we write the above integral in the form

$$
\int_{a}^{b} g(t) d Y_{t} .
$$

Let $Y=\left(Y_{t}, t \geq 0\right)$ be a zero mean process with an absolutely continuous covariance function. We assume that there exists a function $\kappa: \mathbb{R} \times \mathbb{R} \rightarrow \mathbb{R}$ such that

2000 Mathematics Subject Classification: 60G20, 45R05.

Key words and phrases: quasi-diffusion process, stochastic differential equation. 


$$
E\left[\left(Y_{t_{r}}-Y_{t_{r-1}}\right)\left(Y_{t_{s}}-Y_{t_{s-1}}\right)\right]=\int_{t_{r-1}}^{t_{r}} \int_{t_{s-1}}^{t_{s}} \kappa(u, v) d u d v
$$

for all $r \leq s, t_{r} \leq t_{s}, r, s=1, \ldots, n$ and fixed $\mathrm{n}$. The function $\kappa$ may also be a Dirac delta function. We call $\kappa$ a quasi spectral density.

To define the integral $\int_{a}^{b} g(t) d Y_{t}$ consider first the case when $g$ is piecewise constant, i.e. there exist $t_{0}<t_{1}<\cdots<t_{n}$ such that

$$
g(t)=g_{k} \quad \text { for } t_{k-1} \leq t<t_{k}, k=1, \ldots, n,
$$

where the $g_{k}$ are constants, $t_{0}=a, t_{n}=b$. Let

$$
\Delta_{k} Y=Y_{t_{k}}-Y_{t_{k-1}} \text {. }
$$

Then

$$
\int_{a}^{b} g(t) d Y_{t}=\sum_{k=1}^{n} g_{k} \Delta_{k} Y
$$

LEMmA 1.1. If $g_{1}$ and $g_{2}$ are piecewise constant functions, then

$$
\begin{aligned}
E\left[\int_{a}^{b} g_{1}(t) d Y_{t} \int_{a}^{b} g_{2}(t) d Y_{t}\right]= & \iint_{S_{1}} g_{1}(u) g_{2}(v) \kappa(u, v) d u d v \\
& +\iint_{S_{2}} g_{1}(v) g_{2}(u) \kappa(u, v) d u d v
\end{aligned}
$$

where $S_{1}=\{(u, v): a<u<b, u \leq v<b\}$ and $S_{2}=\{(u, v): a<v<b, v<$ $u<b\}$.

Proof. Assume that $a=t_{0} \leq t_{1}<\cdots<t_{n}=b$ are such that the functions $g_{1}$ and $g_{2}$ are constant in every $\left(t_{k-1}, t_{k}\right)$ for $k=1, \ldots, n$ and equal respectively to $g_{1 k}, g_{2 k}$. Then

$$
\begin{aligned}
E\left[\int_{a}^{b} g_{1}(t) d Y_{t} \int_{a}^{b} g_{2}(t) d Y_{t}\right]= & E\left[\sum_{k=1}^{n} g_{1 k} \Delta_{k} Y \sum_{k=1}^{n} g_{2 k} \Delta_{k} Y\right] \\
= & E\left[\sum_{1 \leq i \leq n} \sum_{i \leq j \leq n} g_{1 i} g_{2 j}\left(Y_{t_{i}}-Y_{t_{i-1}}\right)\left(Y_{t_{j}}-Y_{t_{j-1}}\right)\right. \\
& \left.+\sum_{1<i \leq n} \sum_{1 \leq j<i} g_{1 i} g_{2 j}\left(Y_{t_{i}}-Y_{t_{i-1}}\right)\left(Y_{t_{j}}-Y_{t_{j-1}}\right)\right] \\
= & \sum_{1 \leq i \leq n} \sum_{i \leq j \leq n} g_{1 i} g_{2 j} E\left[\left(Y_{t_{i}}-Y_{t_{i-1}}\right)\left(Y_{t_{j}}-Y_{t_{j-1}}\right)\right] \\
& +\sum_{1<i \leq n} \sum_{1 \leq j<i} g_{1 i} g_{2 j} E\left[\left(Y_{t_{i}}-Y_{t_{i-1}}\right)\left(Y_{t_{j}}-Y_{t_{j-1}}\right)\right]
\end{aligned}
$$




$$
\begin{aligned}
& =\sum_{1 \leq i \leq n} \sum_{i \leq j \leq n} g_{1 i} g_{2 j} \int_{t_{i-1}}^{t_{i}} \int_{t_{j-1}}^{t_{j}} \kappa(u, v) d u d v \\
& \quad+\sum_{1 \leq j<n} \sum_{j<i \leq n} g_{1 i} g_{2 j} \int_{t_{j-1}}^{t_{j}} \int_{t_{i-1}}^{t_{i}} \kappa(u, v) d v d u \\
& =\iint_{S_{1}} g_{1}(u) g_{2}(v) \kappa(u, v) d u d v+\iint_{S_{2}} g_{1}(v) g_{2}(u) \kappa(u, v) d u d v .
\end{aligned}
$$

LemMA 1.2. If $\left(Y_{t}, t \geq 0\right)$ is a Gaussian process and $I_{t}=\int_{0}^{t} g(s) d Y_{s}$ with $g$ piecewise constant, then $\left(I_{t}, t \geq 0\right)$ is also Gaussian.

Proof. This is obvious.

We now approximate $g$ by piecewise constant functions. Let $g$ be a function such that

$$
\int_{a}^{b} \int_{a}^{b} g(u) g(v) \kappa(u, v) d u d v<\infty
$$

There exists a sequence $\left\{g_{n}\right\}$ of piecewise constant functions [2] such that $\lim _{n, m \rightarrow \infty} \int_{a}^{b} \int_{a}^{b}\left(g(u)-g_{n}(u)\right)\left(g(v)-g_{m}(v)\right) \kappa(u, v) d u d v=0, \quad n, m=0,1, \ldots$ Then we define the integral $\int_{a}^{b} g(t) d Y_{t}$ as

$$
\int_{a}^{b} g(t) d Y_{t}=\lim _{n \rightarrow \infty} \int_{a}^{b} g_{n}(t) d Y_{t},
$$

the mean square limit of the integrals $\int_{a}^{b} g_{n}(t) d Y_{t}$.

One can prove [2] that this limit does not depend on the approximating sequence $g_{n}$. Furthermore, the properties of the integral (1.2) are analogous to the properties of integrals of piecewise constant functions. In particular, $\int_{a}^{b} g(t) d Y_{t}$ is a Gaussian process as a limit of Gaussian processes [4].

2. Quasi-diffusion solution of the stochastic differential equation. Let $(\Omega, \mathcal{F}, \mathbb{P})$ be a probability space. Let $Y=\left(Y_{t}, t \geq 0\right)$ be a Gaussian process which satisfies (1.1). Let $X_{0}$ be a random variable, independent of $Y$, whose distribution is absolutely continuous.

LEMMA 2.1. Consider the stochastic differential equation

$$
X_{t}=X_{0}+\int_{0}^{t}\left(A_{s}+B_{s} X_{s}\right) d s+\int_{0}^{t} C_{s} d Y_{s}
$$


where $A_{t}, B_{t}, C_{t}:[0, \infty) \rightarrow \mathbb{R}$ are nonrandom continuous functions of $t$ and $X_{0}$ is an initial random variable. Then

(a) there is a unique solution to (2.1) given by the formula

$$
X_{t}=e^{e_{0}^{t} B_{s} d s}\left[X_{0}+\int_{0}^{t} e^{-\int_{0}^{s} B_{u} d u} A_{s} d s+\int_{0}^{t} e^{-\int_{0}^{s} B_{u} d u} C_{s} d Y_{s}\right],
$$

(b) the covariance of the solution is equal to

$$
\begin{aligned}
& \operatorname{Cov}\left(X_{t_{1}}, X_{t_{2}}\right)=e^{\int_{0}^{t_{1}} B_{s} d s+\int_{0}^{t_{2}} B_{s} d s}\left(E\left[X_{0}-E X_{0}\right]^{2}\right. \\
& \left.+\int_{0}^{t_{1}} \int_{0}^{t_{2}} e^{-\int_{0}^{u} B_{s} d s-\int_{0}^{v} B_{s} d s} C_{u} C_{v} \kappa(u, v) d u d v\right) .
\end{aligned}
$$

Proof. (a) We use the method of successive approximations from [3]. Let $X_{t}^{0}=X_{0}$ for all $t$ and

$$
X_{t}^{n}=X_{0}+\int_{0}^{t}\left(A_{s}+B_{s} X_{s}^{n-1}\right) d s+\int_{0}^{t} C_{s} d Y_{s} \quad(n \geq 1) .
$$

Define

$$
\alpha_{t}=\int_{0}^{t} A_{s} d s, \quad \beta_{t}=\int_{0}^{t} B_{s} d s, \quad J_{t}=\int_{0}^{t} C_{s} d Y_{s}
$$

Let

$$
(L h)(t)=\int_{0}^{t} B_{s} h_{s} d s .
$$

This is a Volterra operator on $L^{2}[0, T]$. Then

$$
X_{t}^{1}=X_{0}\left(1+\beta_{t}\right)+\alpha_{t}+J_{t}
$$

and for any $n \geq 1$,

$$
\begin{aligned}
X_{t}^{n+1}= & X_{0}\left[1+\beta_{t}+\cdots+L^{n} \beta_{t}\right]+\left[\alpha_{t}+L \alpha_{t}+\cdots+L^{n} \alpha_{t}\right] \\
& +\int_{0}^{t}\left[1+\left(\beta_{t}-\beta_{s}\right)+\frac{1}{2 !}\left(\beta_{t}-\beta_{s}\right)^{2}+\cdots+\frac{1}{n !}\left(\beta_{t}-\beta_{s}\right)^{n}\right] C_{s} d Y_{s} .
\end{aligned}
$$

Since $L$ is a Volterra operator,

$$
\begin{gathered}
\alpha_{t}+L \alpha_{t}+\cdots+L^{n} \alpha_{t} \rightarrow(I-L)^{-1} \alpha_{t}, \\
(I-L)^{-1} \alpha_{t}=e^{\beta_{t}} \int_{0}^{t} e^{-\beta_{s}} A_{s} d s \\
{\left[1+\beta_{t}+\cdots+L^{n} \beta_{t}\right] \rightarrow 1+(I-L)^{-1} \beta_{t}=1+e^{\beta_{t}} \int_{0}^{t} e^{-\beta_{s}} B_{s} d s=e^{\beta_{t}} .}
\end{gathered}
$$


Let

$$
f_{n}(t, s)=1+\left(\beta_{t}-\beta_{s}\right)+\frac{1}{2 !}\left(\beta_{t}-\beta_{s}\right)^{2}+\cdots+\frac{1}{n !}\left(\beta_{t}-\beta_{s}\right)^{n} .
$$

Then

$$
E\left[\int_{0}^{t} f_{n}(t, s) C_{s} d Y_{s}-\int_{0}^{t} e^{\beta_{t}-\beta_{s}} C_{s} d Y_{s}\right]^{2} \rightarrow 0 \quad \text { as } n \rightarrow \infty .
$$

Hence a solution $X_{t}$ is given by

$$
X_{t}=e^{\int_{0}^{t} B_{s} d s}\left[X_{0}+\int_{0}^{t} e^{-\int_{0}^{s} B_{u} d u} A_{s} d s+\int_{0}^{t} e^{-\int_{0}^{s} B_{u} d u} C_{s} d Y_{s}\right]
$$

Now we prove uniqueness. Let $X_{t}^{1}$ and $X_{t}^{2}$ be two solutions of (2.1). Then

$$
X_{t}^{1}-X_{t}^{2}=\int_{0}^{t} B_{s}\left(X_{s}^{1}-X_{s}^{2}\right) d s
$$

Because $B_{s}$ is bounded by a constant $b$ on any finite interval, we have

$$
\left|X_{t}^{1}-X_{t}^{2}\right| \leq b \int_{0}^{t}\left|X_{s}^{1}-X_{s}^{2}\right| d s
$$

and by Gronwall's lemma ([4, pp. 287-288])

$$
\left|X_{t}^{1}-X_{t}^{2}\right|=0
$$

which completes the proof of uniqueness.

(b) Now we calculate the covariance:

$$
\begin{aligned}
\operatorname{Cov}\left(X_{t_{1}}, X_{t_{2}}\right)= & E\left(X_{t_{1}}-E X_{t_{1}}\right)\left(X_{t_{2}}-E X_{t_{2}}\right) \\
= & e^{f_{0}^{t_{1}} B_{s} d s+\int_{0}^{t_{2}} B_{s} d s}\left(E\left[X_{0}-E X_{0}\right]^{2}\right. \\
& \left.+E\left[\int_{0}^{t_{1}} e^{-\int_{0}^{s} B_{u} d u} C_{s} d Y_{s} \int_{0}^{t_{2}} e^{-\int_{0}^{s} B_{u} d u} C_{s} d Y_{s}\right]\right) \\
= & e^{t_{0}^{t_{1}} B_{s} d s+\int_{0}^{t_{2}} B_{s} d s}\left(E\left[X_{0}-E X_{0}\right]^{2}+E\left[\int_{0}^{t_{1}} e^{-\int_{0}^{s} B_{u} d u} C_{s} d Y_{s}\right]^{2}\right. \\
& \left.+E\left[\int_{0}^{t_{1}} e^{-\int_{0}^{s} B_{u} d u} C_{s} d Y_{s} \int_{t_{1}}^{t_{2}} e^{-\int_{0}^{s} B_{u} d u} C_{s} d Y_{s}\right]\right)
\end{aligned}
$$




$$
\begin{aligned}
& =e^{\int_{0}^{t_{1}} B_{s} d s+\int_{0}^{t_{2}} B_{s} d s}\left(E\left[X_{0}-E X_{0}\right]^{2}\right. \\
& +\int_{0}^{t_{1} \int_{0}} e^{-\int_{0}^{u} B_{s} d s-\int_{0}^{v} B_{s} d s} C_{u} C_{v} \kappa(u, v) d u d v \\
& \quad+\iint_{S_{1}} e^{-\int_{0}^{u} B_{s} d s-\int_{0}^{v} B_{s} d s} C_{u} C_{v} \kappa(u, v) d u d v \\
& \left.+\iint_{S_{2}} e^{-\int_{0}^{u} B_{s} d s-\int_{0}^{v} B_{s} d s} C_{u} C_{v} \kappa(u, v) d u d v\right),
\end{aligned}
$$

where $S_{1}=\left\{(u, v): 0<u<t_{1}, u+t_{1} \leq v<t_{2}\right\}, S_{2}=\left\{(u, v): t_{1}<v<t_{2}\right.$, $\left.v-t_{1}<u<t_{1}\right\}$. Hence

$$
\begin{aligned}
\operatorname{Cov}\left(X_{t_{1}}, X_{t_{2}}\right)= & e^{t_{0}^{t_{1}} B_{s} d s+\int_{0}^{t_{2}} B_{s} d s}\left(E\left[X_{0}-E X_{0}\right]^{2}\right. \\
& +\int_{0}^{t_{1}} \int_{0}^{t_{1}} e^{-\int_{0}^{u} B_{s} d s-\int_{0}^{v} B_{s} d s} C_{u} C_{v} \kappa(u, v) d u d v \\
& \left.+\int_{0}^{t_{1}} \int_{t_{1}}^{t_{2}} e^{-\int_{0}^{u} B_{s} d s-\int_{0}^{v} B_{s} d s} C_{u} C_{v} \kappa(u, v) d u d v\right) \\
= & e^{t_{0}^{t_{1}} B_{s} d s+\int_{0}^{t_{2}} B_{s} d s}\left(E\left[X_{0}-E X_{0}\right]^{2}\right. \\
& \left.+\int_{0}^{t_{1}} \int_{0}^{t_{2}} e^{-\int_{0}^{u} B_{s} d s-\int_{0}^{v} B_{s} d s} C_{u} C_{v} \kappa(u, v) d u d v\right) .
\end{aligned}
$$

Let $Z=\left(Z_{t}, t \geq 0\right)$ be a Gaussian process, and $X_{0}$ be a random variable with density $f\left(t_{0}, x_{0}\right)$, expected value $m_{X_{0}}=E X_{0}$ and variance $\sigma_{X_{0}}^{2}=$ $\operatorname{Var} X_{0}$. Moreover, let $X_{0}$ and $Z$ be independent.

We put

$$
X_{t}=X_{0} g(t)+Z_{t}
$$

where $g \in C^{1}, g\left(t_{0}\right)=1$.

Let $t_{0}<t_{1}<\cdots<t_{n}, m(t)=E Z_{t}, K_{i j}=K\left(t_{i}, t_{j}\right)=\operatorname{Cov}\left(Z_{t_{i}}, Z_{t_{j}}\right)$, where $i, j=0, \ldots, n$ and $Z_{t_{0}}=0$.

Notice that the equation (2.2) can also be written in the form (2.3).

LEMMA 2.2. For every $n>1$ the conditional distribution

$$
X_{t_{n}} \mid X_{t_{n-1}}, \ldots, X_{t_{0}}
$$

is Gaussian.

Proof. It is easy to verify that the $(n+1)$-dimensional distribution of $\left(X_{t_{0}}, \ldots, X_{t_{n}}\right)$ has the form 


$$
\begin{aligned}
& f_{n+1}\left(t_{0}, \ldots, t_{n}, x_{0}, \ldots, x_{n}\right) \\
& =\frac{1}{(2 \pi)^{n / 2} \sqrt{\mathcal{K}^{(n)}}} \exp \left\{-\frac{1}{2} \sum_{k=1}^{n} \frac{1}{\sigma_{k}^{2}}\left[x_{k}-x_{0} g_{k}-m\left(t_{k}\right)\right.\right. \\
& \left.\left.\quad-\frac{1}{\mathcal{K}_{k k}^{(k)}} \sum_{i=1}^{k-1} \mathcal{K}_{i k}^{(k)}\left(x_{i}-x_{0} g_{i}-m\left(t_{i}\right)\right)\right]^{2}\right\} f\left(t_{0}, x_{0}\right),
\end{aligned}
$$

where $\mathcal{K}^{(n)}=\operatorname{det}\left[k_{i j}\right]_{i, j=1}^{n}, \mathcal{K}_{i j}^{(n)}$ is the cofactor of $k_{i j}$ in the matrix $\left[k_{i j}\right]_{i, j=1}^{n}$, and $\sigma_{k}^{2}=\mathcal{K}_{k k}^{(k)} / \mathcal{K}_{k-1, k-1}^{(k)}$.

The conditional density of $X_{t_{n}} \mid X_{t_{n-1}}, \ldots, X_{t_{0}}$ is

$$
\begin{aligned}
f\left(t_{n}, x_{n} \mid t_{0}, \ldots, t_{n-1}, x_{0}, \ldots, x_{n-1}\right) & =\frac{f_{n+1}\left(t_{0}, \ldots, t_{n}, x_{0}, \ldots, x_{n}\right)}{f_{n}\left(t_{0}, \ldots, t_{n-1}, x_{0}, \ldots, x_{n-1}\right)} \\
= & \frac{\sqrt{\mathcal{K}^{(n-1)}}}{\sqrt{2 \pi} \sqrt{\mathcal{K}^{(n)}}} \exp \left\{-\frac{1}{2 \sigma_{n}^{2}}\left[x_{n}-x_{0} g_{n}-m\left(t_{n}\right)\right.\right. \\
& \left.\left.-\frac{1}{\mathcal{K}_{n n}^{(n)}} \sum_{i=1}^{n-1} \mathcal{K}_{i n}^{(n)}\left(x_{i}-x_{0} g_{i}-m\left(t_{i}\right)\right)\right]^{2}\right\} .
\end{aligned}
$$

Let $t_{0}<t_{1}<\cdots<t_{n+k}, \mathbf{t}_{n}=\left(t_{1}, \ldots, t_{n}\right), \Delta t_{n}=t_{n+1}-t_{n}, \mathbf{x}_{n}=$ $\left(x_{1}, \ldots, x_{n}\right) \in \mathbb{R}^{n}$, and let $A_{1}, \ldots, A_{k}$ be Borel sets in $\mathbb{R}$. We will use the following notations:

$$
\begin{aligned}
& \mathbb{P}\left(X_{t_{n+1}} \in A_{1}, \ldots, X_{t_{n+k}} \in A_{k} \mid X_{t_{1}}, \ldots, X_{t_{n}}\right) \\
&=: \mathbb{P}^{(n)}\left(\mathbf{t}_{n}, X_{t_{1}}, \ldots, X_{t_{n}}, t_{n+1}, \ldots, t_{n+k}, A_{1}, \ldots, A_{k}\right), \\
& \frac{1}{\Delta \mathbf{t}_{n}} \int_{A}\left(x-x_{n}\right)^{i} \mathbb{P}^{(n)}\left(\mathbf{t}_{n}, \mathbf{x}_{n} ; t_{n+1}, d x\right)=: Q_{i}\left(\mathbf{t}_{n+1}, \mathbf{x}_{n}, A\right), \quad i=0,1,2 .
\end{aligned}
$$

Definition 2.1. We say that $X=\left(X_{t}, t \geq 0\right)$ is a quasi-diffusion process [7] if for all $n \geq 1, \varepsilon>0, \mathbf{t}_{n}, \mathbf{x}_{n}$,

$$
\lim _{\Delta t_{n} \rightarrow 0^{+}} Q_{0}\left(\mathbf{t}_{n+1}, \mathbf{x}_{n}, V_{\varepsilon}\left(x_{n}\right)\right)=0
$$

and the following limits exist:

$$
\lim _{\Delta t_{n} \rightarrow 0^{+}} Q_{i}\left(\mathbf{t}_{n+1}, \mathbf{x}_{n}, U_{\varepsilon}\left(x_{n}\right)\right)=a_{i}\left(\mathbf{t}_{n}, \mathbf{x}_{n}\right), \quad i=1,2,
$$

where

$$
U_{\varepsilon}\left(x_{n}\right)=\left\{x:\left|x-x_{n}\right|<\varepsilon\right\}, \quad V_{\varepsilon}\left(x_{n}\right)=\mathbb{R} \backslash U_{\varepsilon}\left(x_{n}\right) .
$$

THEOREM 2.1. The process $\left(X_{t}\right)$ defined by (2.2) is a quasi-diffusion process.

Proof. Condition (2.4) is proved in [7] for Gaussian processes. An analysis of the paper [7] shows that it is also true for conditional Gaussian processes considered in [5]. 
Now we calculate the drift coefficient $a_{1}\left(\mathbf{t}_{n}, \mathbf{x}_{n}\right)$ and the diffusion coefficient $a_{2}\left(\mathbf{t}_{n}, \mathbf{x}_{n}\right)$. We shall write $m(t)=E\left(X_{t}\right)$ and $k_{i j}=K\left(t_{i}, t_{j}\right)=$ $\operatorname{Cov}\left(X_{t_{i}}, X_{t_{j}}\right)$. The expected value of the process $X$ is equal to

$$
m\left(t_{i}\right)=e^{\int_{0}^{t_{i}} B_{s} d s}\left[E X_{0}+\int_{0}^{t_{i}} e^{-\int_{0}^{s} B_{u} d u} A_{s} d s\right] .
$$

Notice that by continuity of the functions $A_{s}, B_{s}, C_{s}$ the following limits exist:

$$
\begin{aligned}
\lim _{\Delta t_{n} \rightarrow 0} \frac{1}{\Delta t_{n}}\left[K\left(t_{i}, t_{n+1}\right)-K\left(t_{i}, t_{n}\right)\right] & :=h\left(t_{i}, t_{n}\right), \\
\lim _{\Delta t_{n} \rightarrow 0} \frac{1}{\Delta t_{n}}\left[K\left(t_{n+1}, t_{n+1}\right)-K\left(t_{n}, t_{n+1}\right)\right] & :=h\left(t_{n}\right) .
\end{aligned}
$$

We shall denote by $K_{i j}^{(n)}$ the cofactor of the corresponding element of the matrix $\left[k_{i j}\right]_{i, j=1}^{n}, K^{(n)}$ the determinant of that matrix, $h_{i j}=h\left(t_{i}, t_{j}\right)$, $h_{n}=h\left(t_{n}\right)$,

$$
L^{(n+1)}=\left|\begin{array}{cccc}
k_{11} & \ldots & k_{1 n} & h_{1 n} \\
\ldots & \ldots & \ldots & \ldots \\
k_{n 1} & \ldots & k_{n n} & h_{n n} \\
k_{n+1,1} & \ldots & k_{n+1, n} & 1
\end{array}\right|,
$$

and $L_{i j}^{(n+1)}$ the cofactor of the corresponding element of $L^{(n+1)}$.

Following [7] we can show that

$$
\begin{aligned}
& a_{1}\left(\mathbf{t}_{n}, \mathbf{x}_{n}\right)=m\left(t_{n}\right)+\frac{1}{K^{(n)}} \sum_{i=1}^{n} L_{n+1, i}^{(n+1)}\left(x_{i}-m\left(t_{i}\right)\right), \\
& a_{2}\left(\mathbf{t}_{n}, \mathbf{x}_{n}\right)=\lim _{\Delta t_{n} \rightarrow 0} \frac{1}{\Delta t_{n}}\left(k_{n+1, n+1}-2 k_{n, n+1}+k_{n n}\right)=h_{n}-h_{n n} .
\end{aligned}
$$

This completes the proof.

EXAMPLE 1. If $Y$ is the Wiener process then $\kappa(u, v)=\delta(u-v)$ is the Dirac delta function. The functions $h\left(t_{i}, t_{n}\right)$ and $h\left(t_{n}\right)$ for the process $X$ have the form

$$
\begin{gathered}
h\left(t_{i}, t_{n}\right)=e^{\int_{0}^{t_{i}} B_{s} d s+\int_{0}^{t_{n}} B_{s} d s} B_{t_{n}}\left(\left[E\left(X_{0}-E X_{0}\right)\right]^{2}+\int_{0}^{t_{i}} e^{-2 \int_{0}^{v} B_{s} d s} C_{v}^{2} d v\right), \\
h\left(t_{n}\right)=1+e^{2 \int_{0}^{t_{n}} B_{s} d s} B_{t_{n}}\left(\left[E\left(X_{0}-E X_{0}\right)\right]^{2}+\int_{0}^{t_{n}} e^{-2 \int_{0}^{v} B_{s} d s} C_{v}^{2} d v\right) .
\end{gathered}
$$

Therefore $a_{1}\left(\mathbf{t}_{n}, \mathbf{x}_{n}\right)$ is of the form (2.6) and $a_{2}\left(\mathbf{t}_{n}, \mathbf{x}_{n}\right)=1$.

Acknowledgements. The authors would like to thank Professor $€$. Stettner for valuable remarks, helpful to improve the first version of this paper. 


\section{References}

[1] Yu. L. Daletskiı̆ and S. N. Paromonova, On a formula from the theory of Gaussian measures and on the estimation of stochastic integrals, Teor. Veroyatnost. i Primenen. 19 (1974), 844-849 (in Russian); English transl.: Theory Probab. Appl. 19 (1974), 812-817.

[2] - - - Stochastic integrals with respect to a normally distributed additive set function, Dokl. Akad. Nauk SSSR 208 (1973), 512-515 (in Russian); English transl.: Soviet Math. Dokl. 14 (1973), 96-100.

[3] G. Kallianpur, Stochastic Filtering Theory, Springer, New York, 1980.

[4] I. Karatzas and S. E. Shreve, Brownian Motion and Stochastic Calculus, Springer, New York, 1988.

[5] R. S. Liptser and A. N. Shiryaev, Statistics of Random Processes I, II, Springer, Berlin, 2001.

[6] M. Loève, Probability Theory I, II, 4th ed., Springer, Berlin, 1977.

[7] A. Plucińska, Remarks on prospective equations, Teor. Veroyatnost. i Primenen. 25 (1980), 350-358.

Faculty of Mathematics and Information Science

Warsaw University of Technology

Pl. Politechniki 1, room 228

00-661 Warszawa, Poland

E-mail: amp@mini.pw.edu.pl

w.szymanski@mini.pw.edu.pl

Received on 21.9.2006;

revised version on 11.4 .2007 
